\title{
Erythropoietin induces the osteogenesis of periodontal mesenchymal stem cells from healthy and periodontitis sources via activation of the p38 MAPK pathway
}

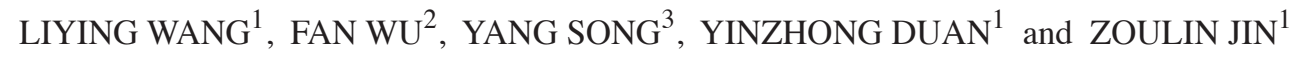 \\ ${ }^{1}$ State Key Laboratory of Military Stomatology and National Clinical Research Center for Oral Diseases and \\ Shaanxi Clinical Research Center for Oral Diseases, Department of Orthodontics, School of Stomatology, \\ The Fourth Military Medical University, Xi'an, Shaanxi 710032; ${ }^{2}$ Laparoscopic Surgery Department, \\ The 451st Hospital of People's Liberation Army; ${ }^{3}$ Department of Stomatology, The 323rd Hospital \\ of People's Liberation Army, Xi'an, Shaanxi 710054, P.R. China
}

Received February 2, 2016; Accepted October 25, 2017

DOI: $10.3892 /$ ijmm.2017.3294

\begin{abstract}
Erythropoietin (Epo), a hematopoietic hormone, has multiple biological functions. Recently, the positively osteogenic effects of Epo on mesenchymal stem cells (MSCs) have attracted broad interest. However, the effects of Epo on the osteogenesis of human periodontal ligament tissue-derived mesenchymal stem cells (hPDLSCs) and periodontitis mesenchymal stem cells (pPDLSCs) from patients with periodontitis remain unknown. In the present study, osteogenic effects of Epo on hPDLSCs and pPDLSCs were investigated, and the results suggested that the effects were mediated by promoting the expression of runt related transcription factor 2, alkaline phosphatase (ALP) and osteocalcin. Using Alizarin Red and ALP staining, it was demonstrated that Epo exerted positive osteogenic effects on hPDLSCs and pPDLSCs. Additionally, Epo upregulated the proliferation of hPDLSCs and pPDLSCs, based on flow cytometric analyses of the cell cycle. To determine the underlying mechanism, the role of the p38 mitogen-activated protein kinase (MAPK) pathway, which is associated with the osteogenesis of hPDLSCs and pPDLSCs, was investigated further. Epo increases p38 phosphorylation (the target of the MAPK pathway) in hPDLSCs and pPDLSCs. Furthermore, when the cells were treated with SB203580, an inhibitor of the p38 MAPK pathway, the osteogenic effects of Epo on hPDLSCs
\end{abstract}

Correspondence to: Professor Yinzhong Duan or Professor Zuolin Jin, State Key Laboratory of Military Stomatology and National Clinical Research Center for Oral Diseases and Shaanxi Clinical Research Center for Oral Diseases, Department of Orthodontics, School of Stomatology, The Fourth Military Medical University, 145 West Changle Road, Xi'an, Shaanxi 710032, P.R. China

E-mail: zuolinj@fmmu.edu.cn

E-mail: duanyz@fmmu.edu.cn

Key words: erythropoietin, human periodontal ligament stem cells, osteogenic differentiation, chronic inflammatory disorder, mitogen-activated protein kinase pathway and pPDLSCs were attenuated. In conclusion, Epo may upregulate the bone formation ability of hPDLSCs and pPDLSCs via the p38 MAPK pathways.

\section{Introduction}

Erythropoietin (Epo) has been established to be an important growth factor that can promote the recruitment mesenchymal stem cells (MSCs) and angiogenesis. Previously, studies have suggested that Epo can trigger bone formation from MSCs. Epo can improve osteogenic differentiation of MSCs in various ways, including by increasing the expression of vascular endothelial growth factor and bone morphogenetic protein 2 (1), and regulating receptor activator of nuclear factor- $\kappa \mathrm{B}$ ligand signaling (2). In vivo, MSCs induced by Epo can facilitate enhanced bone regeneration in a rat alveolar bone defect model and a cranial defect model $(3,4)$.

Periodontitis is a common inflammatory bone disease that can lead to periodontal tissue destruction and tooth loss $(5,6)$. To date, conventional therapies have succeeded in controlling periodontal inflammation but have failed to restore damage of periodontal tissues (7). Previously, tissue engineering based on MSCs has been reported to be an effective approach for periodontal regeneration (8). Human periodontal ligament tissue-derived mesenchymal stem cells (hPDLSCs) are one type of MSCs that can be isolated from periodontal ligament tissue. These cells have multi-directional differentiation capacity and can differentiate into various tissue types, including bone, cartilage, fat and nerves (9-13). Because of the tissue of origin and multi-lineage potential of hPDLSCs, these cells are considered to be a promising cell line for achieving alveolar bone regeneration. Previous studies have established that combining hPDLSCs and biomaterials can achieve partial periodontal regeneration by forming cementum/periodontal ligament-like structures (14-16).

Considering that periodontal hard tissue regeneration is generally the aim of the periodontitis treatment and that periodontal regeneration is difficult to achieve, osteogenic differentiation of hPDLSCs is potentially very important. 
Various factors can impact the osteogenesis of hPDLSCs, among which inflammation is one of the most researched causative factors (17-22). Studies have established that inflammation can inhibit osteogenic differentiation potential via the canonical Wnt and p38 mitogen-activated protein kinase (MAPK) pathways $(21,23)$.

The findings for the current study indicate that Epo can enhance the osteogenesis and proliferation of hPDLSCs and periodontitis mesenchymal stem cells (pPDLSCs). To further investigate the mechanism of these processes, the p38 MAPK pathway was focused on, which has been previously been demonstrated to be important in osteogenic differentiation of hPDLSCs and pPDLSCs (23). Furthermore, previous studies reported that p38 MAPK signaling is activated by Epo (24-26). In the current study, it was demonstrated that Epo can regulate the osteogenic differentiation of hPDLSCs and pPDLSCs via activating the MAPK pathway. When the p38 MAPK signaling was inhibited, the positive effects of Epo on osteogenesis were attenuated. Overall, the findings demonstrate that Epo can enhance bone formation in hPDLSCs and pPDLSCs via the p38 MAPK pathway.

\section{Materials and methods}

Cell culture. Primary hPDLSC cultures were obtained from 10 individuals, 5 male and 5 female, aged 35-45 years, undergoing routine premolar procedures for orthodontic reasons or third molar extractions. For every single experiment, cells from at least 3 different individuals were tested. The pPDLSCs were obtained from 7 individuals, 4 male and 3 female, aged 27-52 years, who were diagnosed with stable phase periodontitis with two-thirds alveolar bone destruction or at least one periodontal pocket (depth, $>5 \mathrm{~mm}$ ). For every single experiment, cells from at least 3 different individuals were tested. None of these selected subjects had recent periodontal infection or systemic disease, a history of smoking or histories of maxillofacial surgery, radiotherapy or chemotherapy. All samples were collected at the Dental Clinic of the Fourth Military Medical University (Xi'an, China). Each participant provided written informed consent, and the study was approved by the Hospital's Ethics Committee (license no. IRB-REV-2015038). The tissue were obtained from the periodontal ligament of the root surface and using type 1 collagenase digestion $(0.66 \mathrm{mg} / \mathrm{ml}$; Sigma-Aldrich; Merck KGaA, Darmstadt, Germany) at $37^{\circ} \mathrm{C}$ for $>20 \mathrm{~min}$. Single-cell suspensions were cultured in $\alpha$-minimum essential medium ( $\alpha$-MEM) (Gibco; Thermo Fisher Scientific, Inc., Waltham, MA, USA) supplemented with $10 \%$ fetal bovine serum (FBS; Gibco; Thermo Fisher Scientific, Inc.), glutamine, penicillin and streptomycin. At passage 3, hPDLSCs and pPDLSCs were isolated using immunomagnetic beads (M2450; Dynal Biotech, Wirral, UK) with STRO-1 antibodies (340106; Biolegend, San Diego, CA, USA), as previously described (19). Cells at passages 3-5 were used in this study.

Epo treatment. hPDLSCs and pPDLSCs at passage 3 ( $1 \times 10^{5} /$ well) were cultured in $\alpha$-MEM (10\% FBS) until they reached $80 \%$ confluence. Then, cells were incubated with Epo medium (Epo medium contains $20 \mathrm{IU} / \mathrm{ml}$ Epo in $\alpha$-MEM with $10 \%$ FBS) and the medium was changed in 2 days.
Subsequently, the cells were cultured in osteogenic-inducing media for 7 or 21 days to induce osteogenic differentiation.

SB203580 treatment. HPDLSCs and pPDLSCs at passage 3 ( $1 \times 10^{5} /$ well) were cultured in $\alpha$-MEM (10\% FBS) until they reached $80 \%$ confluence. Then, cells were incubated with SB203580 medium (SB203580 medium contained $10 \mu \mathrm{mol} / 1 \mathrm{SB} 203580$ in $\alpha$-MEM with $10 \% \mathrm{FBS})$ and the medium was changed in 2 days. Then the cells were cultured in osteogenic-inducing media for 7 or 21 days to induce osteogenic differentiation

Osteogenic differentiation. Cells (1x10 $/$ well) were cultured were cultured until they reached $80 \%$ of the culture flask. Then, media were changed with osteogenic medium (100 nM dexamethasone, $50 \mathrm{mg} / \mathrm{ml}$ ascorbic acid, and $5 \mathrm{mM} \beta$-glycerophosphate; Sigma-Aldrich; Merck KGaA) cells were cultured for 7 or 21 days. The alkaline phosphatase (ALP) activity assay was performed following osteogenic induction for 7 days using an ALP kit according to the manufacturer's instructions (Nanjing Jiancheng Bioengineering Institute, Nanjing, China). ALP staining was performed using a BCIP/NBT ALP Color Development kit according to the manufacturer's instructions (Beyotime Institute of Biotechnology, Haimen, China). Following inducing in osteogenic medium for 21 days, Alizarin Red staining was performed. Cells were washed with $10 \%$ FBS in PBS twice. Then cells were fixed with $60 \%$ isopropanol for $1 \mathrm{~min}$. Subsequently, cells were washed with distilled water for 3 min and stained using 1\% Alizarin Red (Sigma-Aldrich; Merck $\mathrm{KGaA}$ ) at room temperature for $30 \mathrm{~min}$. The Alizarin Red-stained nodules were visualized under an Olympus BX51 light microscope equipped with an Olympus DP70 camera (Olympus, Co., Tokyo, Japan). To quantify Alizarin staining, mineralized nodules were dissolved in $0.5 \mathrm{~N} \mathrm{HCl}$ with $0.5 \mathrm{ml}$ $5 \%$ SDS for $30 \mathrm{~min}$.

To quantify Alizarin Red-stained nodules, the stain was solubilized with $0.5 \mathrm{ml} 5 \% \mathrm{SDS}$ in $0.5 \mathrm{~N} \mathrm{HCl}$ for $30 \mathrm{~min}$ at room temperature. Subsequently, $0.15 \mathrm{ml}$ of the liquid was transferred to a 96-well plates and absorbance value were measured at $405 \mathrm{~nm}$ using a microplate reader (Bio-Tek Instruments, Winooski, VT, USA). All assays were repeated three times.

Western blot analyses. hPDLSCs and pPDLSCs were lysed in radioimmunoprecipitation assay buffer and protein content of the lysate was determined using a protein assay kit (Beyotime Institute of Biotechnology) according to the manufacturer's instructions. Then, $20 \mathrm{mg}$ cell protein lysate was boiled for $10 \mathrm{~min}$ and was resolved using 10\% SDS-PAGE. The proteins were transferred to a polyvinylidene difluoride membrane (Bio-Rad Laboratories, Inc., Hercules, CA, USA). Membranes were blocked in $5 \%$ bovine serum albumin (Solarbio, Beijing, China) at room temperature for $2 \mathrm{~h}$ and were then incubated with primary antibodies at room temperature for $4 \mathrm{~h}$. Subsequently, membranes were incubated with anti-rabbit or anti-mouse $\operatorname{IgG}$ antibodies at room temperature for $2 \mathrm{~h}$. Immunodetection was performed using the Western-Light Chemiluminescent Detection system (JS-1070P; Peiqing P\&Q Science and Technology, Shanghai, China). We then performed densitometry using Image J software (National 
Institutes of Health, Bethesda, MD, USA). All assays were repeated three times.

The following primary antibodies were used: p38 (1:1,000; cat. no. 9212S) and phospho (p)-p38 (1:1,000; cat. no. 4511; both from Cell Signaling Technology, Inc.); $\beta$-actin (1:800; cat. no. CW0096A; CWBio, Co., Ltd., Beijing, China); secondary antibodies, anti-rabbit and anti-mouse IgG antibodies (1: 10,000; cat. nos. 115-035-003 and 111-035-003; Jackson ImmunoResearch Laboratories, Inc., West Grove, PA, USA)

Reverse transcription-quantitative polymerase chain reaction $(R T-q P C R)$. Total RNA was isolated using TRIzol reagent (Life Technologies; Thermo Fisher Scientific, Inc.) according to the manufacturer's instructions and converted into cDNA using a PrimeScript RT reagent kit (Takara Biotechnology Co., Ltd., Dalian, China). For RT of mRNA, random-primed cDNA was synthesized from $2 \mathrm{mg}$ total RNA. qPCR analysis was performed using the SYBR Premix Ex Taq II kit (Takara Biotechnology Co., Ltd.) and detected on the ABI Prism 7500 HT sequence detection system (Applied Biosystems; Thermo Fisher Scientific, Inc.). $\beta$-actin was used for quantitation of mRNAs. The data were analyzed using the $2^{-\Delta \Delta \mathrm{Cq}}(27)$ relative expression method. All assays were repeated three times. Primer pairs were as follows: Runt related transcription factor 2 (Runx2), 5'-CCCGTGGCCTTCAAGGT-3', 5'-CGTTACCCGCCATGACAGTA-3'; ALP, 5'-GGACCA TTCCCACGTCTTCAC-3', 5'-CCTTGTAGCCAGGCC CATTG-3'; osteocalcin (OCN), 5'-CCCAGGCGCTACCTG TATCAA-3', 5'-GGTCAGCCAACTCGTCACAGTC-3'; and $\beta$-actin, 5'-TGGCACCCAGCACAATGAA-3', 5'-CTAAGT CATAGTCCGCCTAGAAGCA-3'.

Flow cytometric analysis of the cell cycle. Cell cycle was analyzed by measuring the amount of propidium iodide (PI) in ethanol fixed cells. Cells were routine cultured for 5 days. Cells $\left(2 \times 10^{5}\right.$ cells) were washed with PBS three times and fixed with cold $70 \%$ ethanol for $24 \mathrm{~h}$. Then, cells were washed with PBS three times and resuspended in $1 \mathrm{ml}$ permeabilizing solution (Triton X-100, sodium azide $0.01 \%$ and RNase A $100 \mu \mathrm{g} / \mu \mathrm{l}$ ) in PBS for $10 \mathrm{~min}$. Following one wash in PBS, cells were stained with $1 \mathrm{ml}$ PBS with PI $(2.5 \mathrm{mg} / \mathrm{ml})$ and incubated for $15 \mathrm{~min}$ at $4^{\circ} \mathrm{C}$. Finally, the cell cycle was measured using a flow cytometer. Cells in $\mathrm{G}_{2}$ and $\mathrm{S}$ phases were considered to be in the proliferation phase. All assays were repeated three times.

Statistical analyses. All experiments in this study were repeated at least three times. Data were analyzed using an independent samples t-test, and presented as the mean \pm standard deviation. The Bonferroni correction was applied for multiple comparisons. $\mathrm{P}<0.05$ was considered to indicate a statistically significant difference.

\section{Results}

Effect of Epo on the proliferation of hPDLSCs and pPDLSCs. hPDLSCs and pPDLSCs were cultured in normal culture medium and Epo-induced culture medium (with $20 \mathrm{IU} / \mathrm{ml}$ Epo). Cell proliferation capacities were assessed using cell cycle analysis. The percentages of cells in the
A
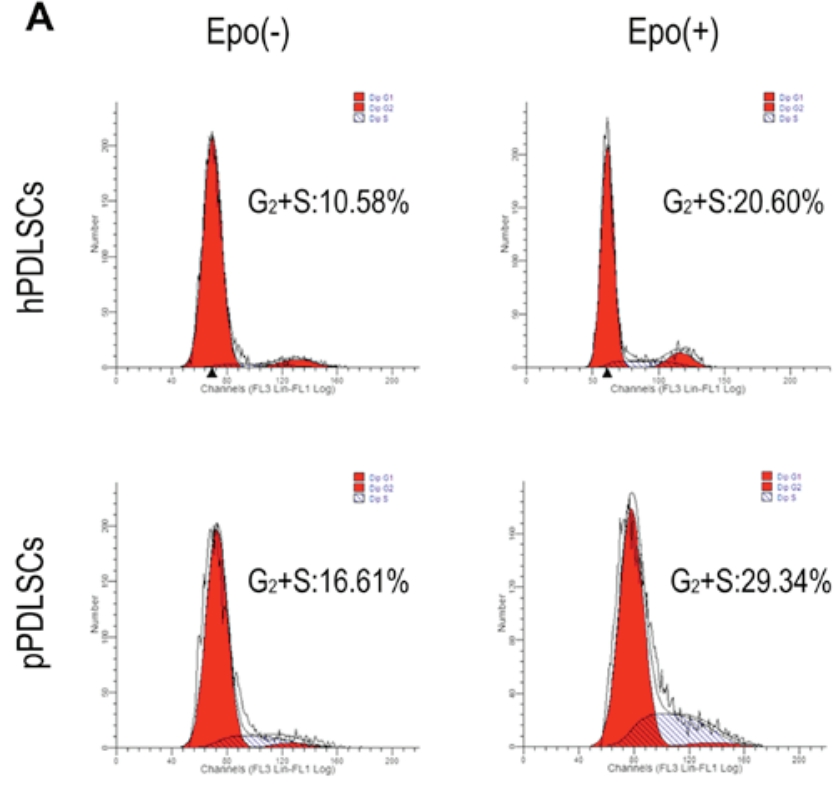

B
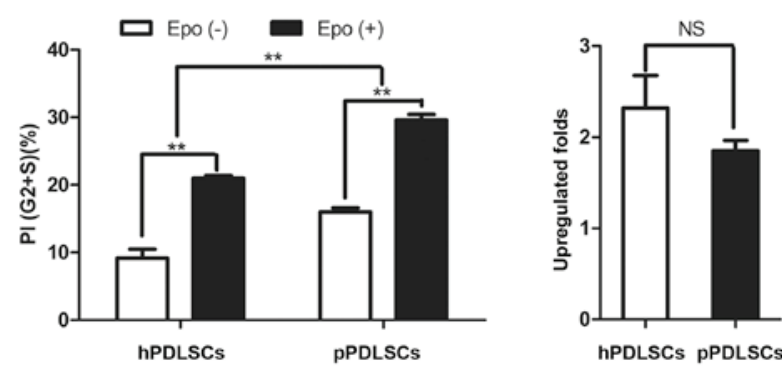

Figure 1. Effects of Epo on the proliferation of hPDLSCs and pPDLSCs. (A) Percentage of cells in $\mathrm{G}_{2}+\mathrm{S}$ phase was measured by flow cytometry at day 5. (B) Quantitative data of the cell cycle analysis, PI of hPDLSCs and pPDLSCs with or without Epo induced (left) and fold upregulation (right) of PI in hPDLSCs and pPDLSCs induced by Epo. Data represent mean \pm standard deviation. ${ }^{* *} \mathrm{P}<0.01$. Epo(-), cells cultured without Epo; Epo(+), cells cultured with Epo; Epo, erythropoietin; hPDLSC, human periodontal ligament tissue-derived mesenchymal stem cells; pPDLSCs, periodontitis mesenchymal stem cells; PI, proliferation index; NS, not significant.

proliferation phase were considered as the proliferation index (PI). The PI of hPDLSCs and pPDLSCs induced by Epo was increased compared with untreated hPDLSCs and pPDLSCs (Fig. 1A). Notably, regarding proliferation, hPDLSCs exhibited greater sensitivity to Epo based on the PI; however, pPDLSCs exhibited greater proliferation ability than hPDLSCs (Fig. 1B).

Effects of Epo on osteogenic differentiation of hPDLSCs and pPDLSCs. The effects of Epo on the osteogenic differentiation of hPDLSCs and pPDLSCs were analyzed by ALP staining (Fig. 2A) and ALP activity assay (Fig. 2B), and the mRNA transcript levels of genes Runx2, ALP and OCN (Fig. 2C) were measured. Furthermore, to characterize the effect of Epo, mineralized nodule formation was analyzed by Alizarin Red staining following induction of cells in osteogenic media for 21 days. Epo promoted the osteogenesis of hPDLSCs and pPDLSCs (Fig. 2D and E). 


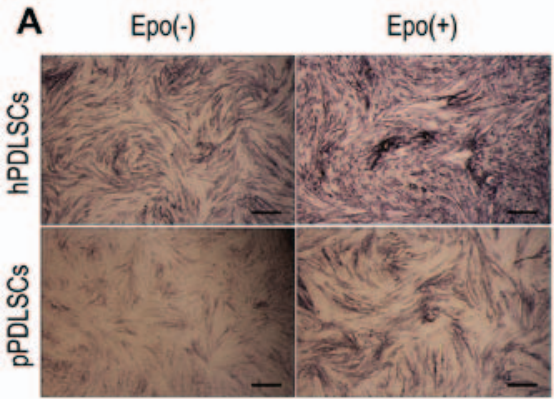

B
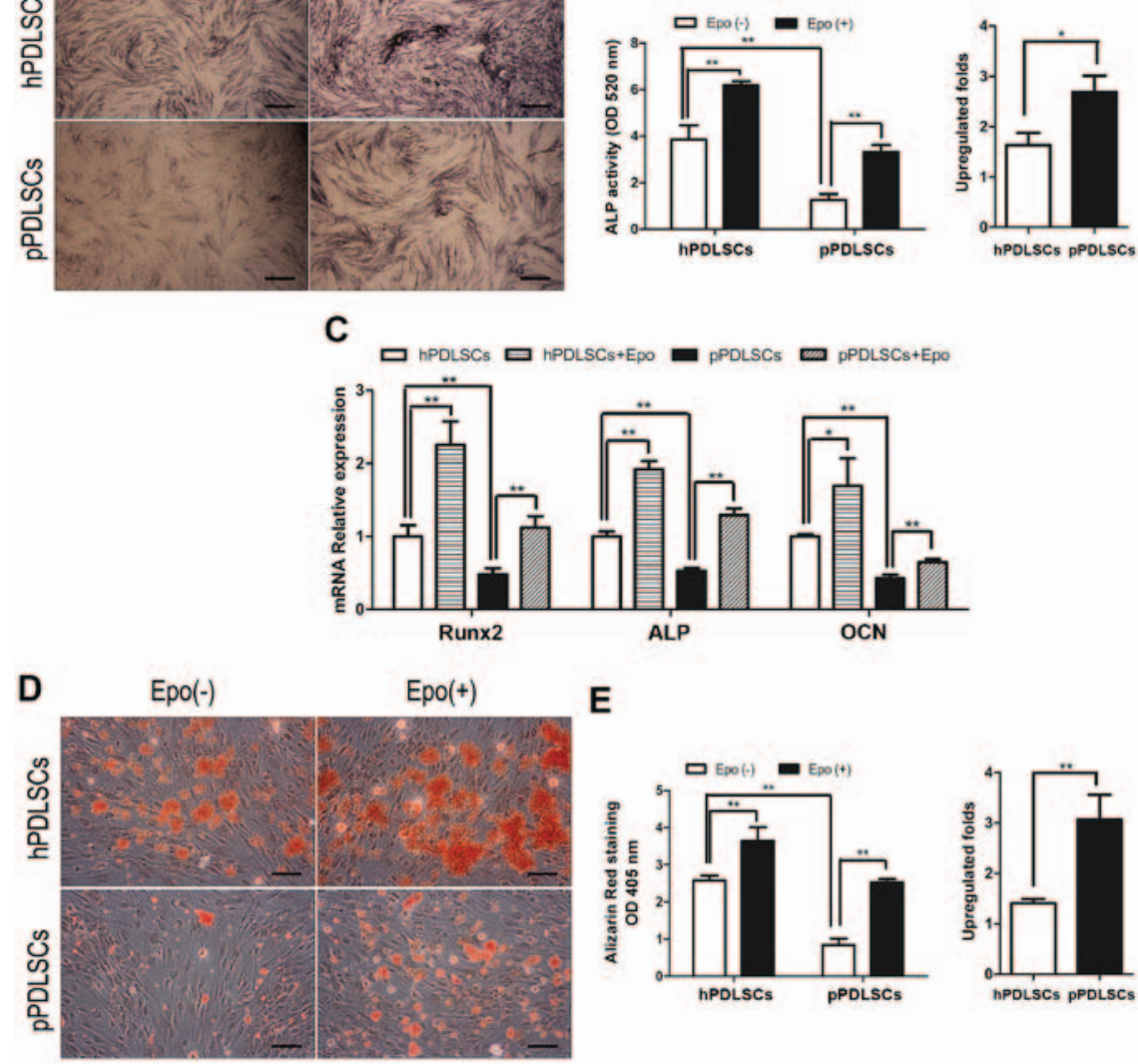

E
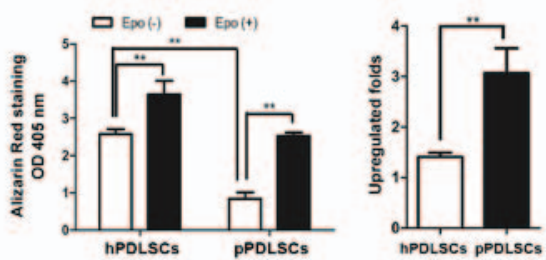

Figure 2. Effects of Epo on the osteogenic differentiation of hPDLSCs and pPDLSCs. (A) Osteogenic differentiation was determined by ALP staining at day 7 after osteogenic differentiation induced. (B) ALP activity was measured by ALP activity assay at day 7 after osteogenic differentiation induced and fold upregulation of ALP activity in hPDLSCs and pPDLSCs induced by Epo. (C) Expression levels of the osteogenic genes ALP, Runx2 and OCN were measured by reverse transcription-quantitative polymerase chain reaction at day 7 after osteogenic differentiation induced. (D) Osteogenic differentiation was determined by Alizarin Red S staining at day 21 after osteogenic differentiation induced. (E) Calcium concentration determined by Alizarin Red S and fold upregulation of calcium concentration in hPDLSCs and pPDLSCs induced by Epo. Data are presented as the mean \pm standard deviation. $\mathrm{P}<0.05,{ }^{* *} \mathrm{P}<0.01$. Scale bar, $100 \mu \mathrm{m}$. Epo(-), cells cultured without Epo; Epo(+), cells cultured with Epo; Epo, erythropoietin; hPDLSC, human periodontal ligament tissue-derived mesenchymal stem cells; pPDLSCs, periodontitis mesenchymal stem cells; Runx2, runt related transcription factor 2; ALP, alkaline phosphatase; OCN, osteocalcin; OD, optical density.

A

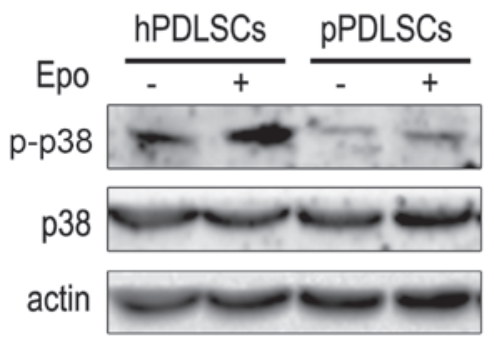

B

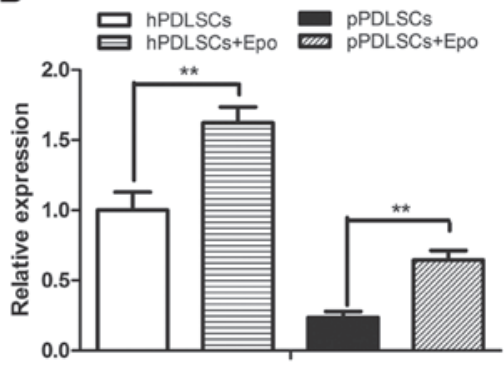

Figure 3. Effects of Epo on the p38 mitogen-activated protein kinase pathway. (A) p-p38 level was examined by western blot analysis and (B) scanning densitometry. Actin was used as the internal control. All tests were measured 7 days after cells cultured. Data are presented as the mean \pm standard deviation. ${ }^{* *} \mathrm{P}<0.01$. hPDLSC, human periodontal ligament tissue-derived mesenchymal stem cells; pPDLSCs, periodontitis mesenchymal stem cells; Epo, erythropoietin; p-p38, phospho-p38.

We also found that inflammation could affect osteogenesis in hPDLSCs (Fig. 2A-E).

Effects of Epo on the p38 MAPK pathways. To identify the mechanism by which Epo regulates the bone formation capacity of hPDLSCs and pPDLSCs, the effects of Epo on the $\mathrm{p} 38$ MAPK pathways in hPDLSCs and pPDLSCs were analyzed based on the phosphorylation of p38. hPDLSCs and pPDLSCs induced by Epo exhibited increased p-p38 level compared with the untreated control groups (Fig. 3). However, the expression of total $\mathrm{p} 38$ protein was not altered.

Role of p38 MAPK pathways in Epo-mediated regulation of osteogenic differentiation of hPDLSCs and pPDLSCs. 
A

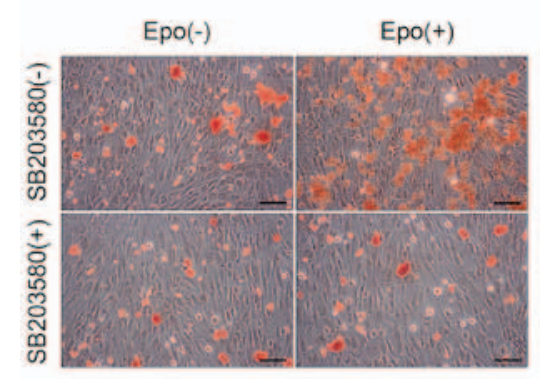

B

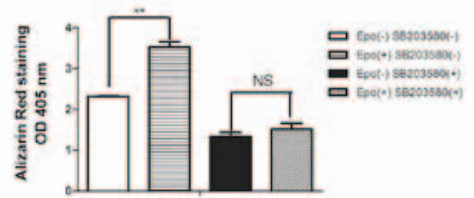

C

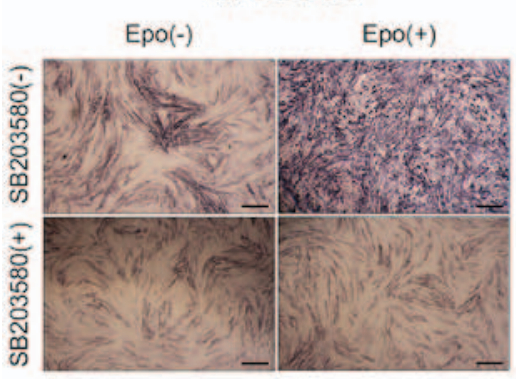

D

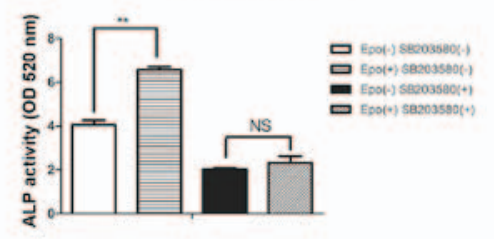

E

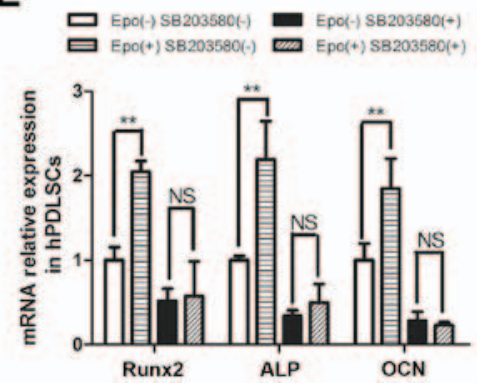

pPDLSCs

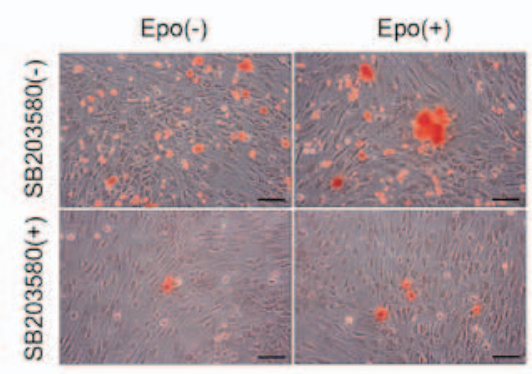

pPDLSCs

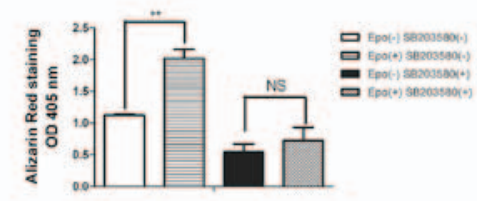

pPDLSCs

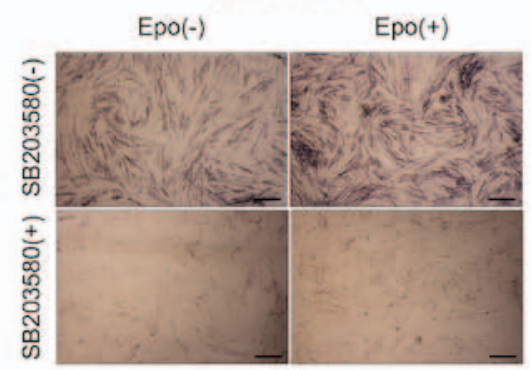

pPDLSCs
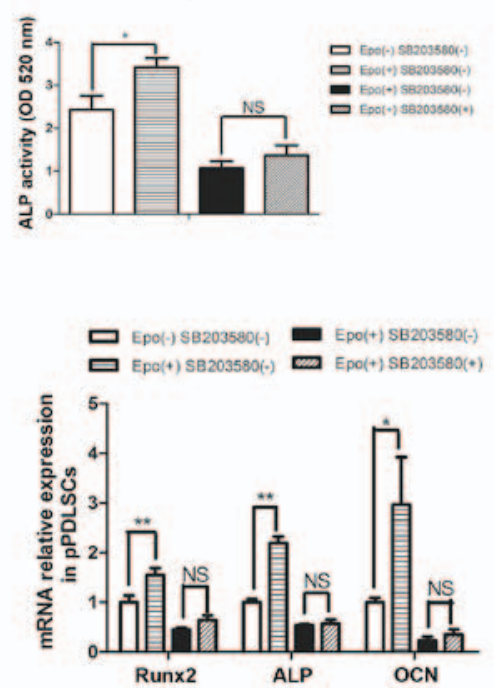

Figure 4. Effects of the p38 mitogen-activated protein kinase pathway on the processes of Epo regulating the osteogenic differentiation of hPDLSCs and pPDLSCs. (A) Osteogenic differentiation was determined by Alizarin Red S staining at day 21 after osteogenic differentiation induced. (B) Alizarin Red S staining was used to determine calcium levels. (C) Osteogenic differentiation was determined by alkaline phosphatase (ALP) staining at day 7 after osteogenic differentiation induced. (D) ALP activity was measured by ALP activity assay at day 7 after osteogenic differentiation induced. (E) Expression levels of the osteogenic genes ALP, Runx2 and OCN were measured by reverse transcription-quantitative polymerase chain reaction at day 7 after osteogenic differentiation induced. All all analyses were performed after 7 days in culture. Data are presented as the mean \pm standard deviation. ${ }^{*} \mathrm{P}<0.05,{ }^{* *} \mathrm{P}<0.01$. Scale bar, $100 \mu \mathrm{m}$. hPDLSC, human periodontal ligament tissue-derived mesenchymal stem cells; pPDLSCs, periodontitis mesenchymal stem cells; Epo, erythropoietin; Epo(-), cells cultured without Epo; Epo(+), cells cultured with Epo; SB203580(-), cells cultured without SB203580; SB203580(+), cells cultured with SB203580; ALP, alkaline phosphatase; OD, optical density; NS, not significant; Runx2, runt related transcription factor 2; OCN, osteocalcin.

To characterize the role of the p38 MAPK pathways on the processes whereby Epo regulates the osteogenic differentiation of hPDLSCs and pPDLSCs, this pathway was inhibited using the pathway-specific inhibitor SB203580. Following treatment with SB203580, the osteogenic capacities of hPDLSCs and pPDLSCs with or without Epo were determined. The mineralized nodule formation (Fig. 4A and B) and ALP activity (Fig. 4C and D) induced by Epo were also decreased 
significantly when cells were co-treated with SB203580. The expression level of osteogenic genes was measured using RT-qPCR. SB203580 treatment led to a significant decrease in Epo-induced Runx2, ALP and OCN expression (Fig. 4E). In conclusion, the effects of Epo on the osteogenic differentiation of hPDLSCs and pPDLSCs were reduced when the MAPK pathway was inhibited by SB203580.

\section{Discussion}

Epo, which was first discovered as a regulator of erythropoiesis, has been used as a therapeutic for certain red blood cell disorders (28). Furthermore, it has been reported to have multiple biological functions, including repair of neuronal injury, improve the proliferation and differentiation in endothelial progenitor cells, and promotion of wound healing (29). Recently, the effects of Epo on the regulation of osteogenic differentiation of MSCs have attracted significant attention. Epo may induce the osteogenesis of MSCs by promoting cell proliferation, migration and differentiation (30). Kim et al (3) reported that Epo can regulate differentiation of both osteoblasts and osteoclasts through mechanistic target of rapamycin kinase (mTOR) signaling. In this process, Epo improved bone formation of MSCs. Furthermore, Epo also increases nuclear factor of activated T-cells cytoplasmic 1 expression and decreases cathepsin $\mathrm{K}$ expression in an mTOR-independent manner, resulting in an increase of osteoclast numbers and a decrease in resorption activity (3). However, the effects of Epo on osteogenic differentiation of hPDLSCs remain unknown.

In the present study, Epo increased the osteogenic differentiation of hPDLSCs, as indicated by the expression of osteogenic genes, Alizarin Red and ALP staining, and an ALP activity assay. Osteogenic differentiation was defined based on the differentiation of a sufficient number of cells. Thus, the effect of Epo on the proliferation of hPDLSCs was also assessed, which was determined by cell cycle analyses. Epo increased the proliferation of hPDLSCs.

Periodontitis is a type of inflammatory disease characterized by the destruction of periodontal tissues that contain alveolar bone, periodontal ligament and root cementum. However, the regeneration of periodontal hard tissues is always an essential problem in such studies.

It has previously been established that hPDLSCs present in an inflammatory microenvironment for a long period will exhibit negative effects on osteogenesis (23). Such exposure may lead to periodontal bone tissue defects. Therefore, determining how to restore the normal capacity of osteogenic differentiation in pPDLSCs is an important goal for studies of periodontal regeneration. In the present study, Epo upregulated the osteogenesis of pPDLSCs by inducing the expression of osteogenic genes. Alizarin Red and ALP staining, and ALP activity were also increased by Epo. In addition, among the osteogenesis genes (Runx2, ALP and OCN), Runx2 was changed most markedly. Runx 2 is an osteoblast differentiation factor that is often expressed in mesenchymal cell types. Runx 2 often promotes the level of bone morphogenic protein and expressed in terminally differentiated osteoblasts (31). Notably, proinflammatory T cells may inhibit MSC-mediated bone formation via tumor necrosis factor (TNF)- $\alpha$-induced downregulation of Runx2 (32). Therefore, the promotion of bone regeneration by Epo-induced pPDLSCs may exert an anti-inflammatory effect that reduces levels of TNF- $\alpha$ expression. Compared with hPDLSCs, pPDLSCs have increased capacities for proliferation. We also demonstrated that the proliferation of pPDLSCs was further improved when induced by Epo.

The MAPK pathway is involved in various cellular processes, including cell proliferation, survival and differentiation (33-35). Among the mediators of the MAPK pathway, p38 was reported to be involved in early and late bone formation of osteoblasts, MSCs and MC3T3-E1 cells (36). In this process, $\mathrm{p} 38$ could increase bone homeostasis and osteogenesis through Runx2, the key transcription factor of osteogenic differentiation (37). Furthermore, the findings of the current study indicate that the p38 MAPK pathway may also promote bone regeneration of hPDLSCs and pPDLSCs. Chang et al (38) reported that activating p38 MAPK signaling can enhance the bone formation ability of hPDLSCs. When the p38 level was reduced, the osteogenic capacities of hPDLSCs were inhibited (39). In an inflammatory environment, the activation of p38 MAPK was altered in PDLSCs during the osteogenic differentiation, and the osteogenesis ability of pPDLSCs was damaged (23).

The present study identified that the p38 phosphorylation level was decreased in pPDLSCs, compared with hPDLSCs, which is consistent with a previous study (23). Additionally, the results demonstrated that Epo promoted the phosphorylation of p38, which demonstrated that $\mathrm{p} 38$ MAPK can be activated by Epo in hPDLSCs and pPDLSCs. Previous studies have demonstrated that Epo can positively regulate the p38 MAPK pathway in multiple cell lines, including smooth muscle cells, heart cells and MSCs (24-26). However, whether this process is involved in osteogenesis has not been thoroughly investigated. In the present study, the osteogenic effects of Epo in hPDLSCs and pPDLSCs were attenuated when the $\mathrm{p} 38$ signaling pathway was inhibited. All these findings strongly demonstrate that Epo can induce osteogenesis of hPDLSCs and pPDLSCs by activating the p38 MAPK pathway.

In conclusion, the present study established that Epo upregulates osteogenesis and the proliferation of hPDLSCs and pPDLSCs. The underlying mechanism may involve p38 MAPK signaling. Further animal studies are required to verify the function and safety of Epo in promoting the osteogenesis capacity of hPDLSCs and pPDLSCs in vivo.

\section{Acknowledgements}

This study was supported by grants from the National Natural Science Foundation of China (grant no. 81271176)

\section{References}

1. Holstein JH, Orth M, Scheuer C, Tami A, Becker SC, Garcia P, Histing T, Mörsdorf P, Klein M, Pohlemann T, et al: Erythropoietin stimulates bone formation, cell proliferation, and angiogenesis in a femoral segmental defect model in mice. Bone 49: 1037-1045, 2011.

2. Shiozawa Y, Jung Y, Ziegler AM, Pedersen EA, Wang J, Wang Z, Song J, Wang J, Lee CH, Sud S, et al: Erythropoietin couples hematopoiesis with bone formation. PLoS One 5: e10853, 2010.

3. Kim J, Jung Y, Sun H, Joseph J, Mishra A, Shiozawa Y, Wang J, Krebsbach PH and Taichman RS: Erythropoietin mediated bone formation is regulated by mTOR signaling. J Cell Biochem 113: 220-228, 2012 
4. Chen S, Li J, Peng H, Zhou J and Fang H: Administration of erythropoietin exerts protective effects against glucocorticoid-induced osteonecrosis of the femoral head in rats. Int J Mol Med 33: 840-848, 2014

5. Pihlstrom BL, Michalowicz BS and Johnson NW: Periodontal diseases. Lancet 366: 1809-1820, 2005.

6. Nanci A and Bosshardt DD: Structure of periodontal tissues in health and disease. Periodontol 40: 11-28, 2006.

7. Chen FM, Zhang J, Zhang M, An Y, Chen F and Wu ZF: A review on endogenous regenerative technology in periodontal regenerative medicine. Biomaterials 31: 7892-7927, 2010.

8. Izumi Y, Aoki A, Yamada Y, Kobayashi H, Iwata T, Akizuki T, Suda T, Nakamura S, Wara-Aswapati N, Ueda M, et al: Current and future periodontal tissue engineering. Periodontol 56 166-187, 2011.

9. Seo BM, Miura M, Gronthos S, Bartold PM, Batouli S, Brahim J, Young M, Robey PG, Wang CY and Shi S: Investigation of multipotent postnatal stem cells from human periodontal ligament. Lancet 364: 149-155, 2004.

10. Akiyama K, Chen C, Gronthos S and Shi S: Lineage differentiation of mesenchymal stem cells from dental pulp, apical papilla, and periodontal ligament. Methods Mol Biol 887: 111-121, 2012.

11. Washio K, Iwata T, Mizutani M, Ando T, Yamato M, Okano T and Ishikawa I: Assessment of cell sheets derived from human periodontal ligament cells: a pre-clinical study. Cell Tissue Res 341: 397-404, 2010.

12. Gault P, Black A, Romette JL, Fuente F, Schroeder K, Thillou F, Brune T, Berdal A and Wurtz T: Tissue-engineered ligament: implant constructs for tooth replacement. J Clin Periodontol 37: 750-758, 2010

13. Yang $\mathrm{H}$, Gao LN, An Y, Hu CH, Jin F, Zhou J, Jin Y and Chen FM: Comparison of mesenchymal stem cells derived from gingival tissue and periodontal ligament in different incubation conditions. Biomaterials 34: 7033-7047, 2013.

14. Ma Z, Li S, Song Y, Tang L, Ma D, Liu B and Jin Y: The biological effect of dentin noncollagenous proteins (DNCPs) on the human periodontal ligament stem cells (HPDLSCs) in vitro and in vivo. Tissue Eng Part A 14: 2059-2068, 2008

15. Chen FM, Zhao YM, Wu H, Deng ZH, Wang QT, Zhou W, Liu Q, Dong GY, Li K, Wu ZF, et al: Enhancement of periodontal tissue regeneration by locally controlled delivery of insulin-like growth factor-I from dextran-co-gelatin microspheres. J Control Release 114: 209-222, 2006.

16. Ramseier CA, Abramson ZR, Jin Q and Giannobile WV: Gene therapeutics for periodontal regenerative medicine. Dent Clin North Am 50: 245-263, 2006.

17. Wu RX, Bi CS, Yu Y, Zhang LL and Chen FM: Age-related decline in the matrix contents and functional properties of human periodontal ligament stem cell sheets. Acta Biomater 22: 70-82, 2015.

18. Zhang J, An Y, Gao LN, Zhang YJ, Jin Y and Chen FM: The effect of aging on the pluripotential capacity and regenerative potential of human periodontal ligament stem cells. Biomaterials 33: 6974-6986, 2012.

19. Zhou Y,Fan W and Xiao Y: The effect of hypoxia on the stemness and differentiation capacity of PDLC and DPC. BioMed Res Int 2014: 890675, 2014

20. Kim SY, Kang KL, Lee JC and Heo JS: Nicotinic acetylcholine receptor $\alpha 7$ and $\beta 4$ subunits contribute nicotine-induced apoptosis in periodontal ligament stem cells. Mol Cells 33: 343-350, 2012.

21. Liu W, Konermann A, Guo T, Jäger A, Zhang L and Jin Y: Canonical Wnt signaling differently modulates osteogenic differentiation of mesenchymal stem cells derived from bone marrow and from periodontal ligament under inflammatory conditions. Biochim Biophys Acta 1840: 1125-1134, 2014.

22. Zheng W, Wang S, Wang J and Jin F: Periodontitis promotes the proliferation and suppresses the differentiation potential of human periodontal ligament stem cells. Int J Mol Med 36: 915-922, 2015

23. Liu N, Shi S, Deng M, Tang L, Zhang G, Liu N, Ding B, Liu W, Liu Y, Shi $\mathrm{H}$, et al: High levels of $\beta$-catenin signaling reduce osteogenic differentiation of stem cells in inflammatory microenvironments through inhibition of the noncanonical Wnt pathway. J Bone Miner Res 26: 2082-2095, 2011.
24. Liu N, Tian J, Cheng J and Zhang J: Effect of erythropoietin on the migration of bone marrow-derived mesenchymal stem cells to the acute kidney injury microenvironment. Exp Cell Res 319: 2019-2027, 2013

25. Park SL, Won SY, Song JH, Kambe T, Nagao M, Kim WJ and Moon SK: EPO gene expression promotes proliferation, migration and invasion via the p38MAPK/AP-1/MMP-9 pathway by 21 WAF1 expression in vascular smooth muscle cells. Cell Signal 27: 470-478, 2015.

26. Rafiee P, Shi Y, Su J, Pritchard KA Jr, Tweddell JS and Baker JE: Erythropoietin protects the infant heart against ischemia-reperfusion injury by triggering multiple signaling pathways. Basic Res Cardiol 100: 187-197, 2005.

27. Livak KJ and Schmittgen TD: Analysis of relative gene expression data using real-time quantitative PCR and the 2(-Delta Delta C(T)) Method. Methods 25: 402-408, 2001.

28. Wu H, Liu X, Jaenisch R and Lodish HF: Generation of committed erythroid BFU-E and CFU-E progenitors does not require erythropoietin or the erythropoietin receptor. Cell 83: 59-67, 1995

29. Broxmeyer HE: Erythropoietin: multiple targets, actions, and modifying influences for biological and clinical consideration. J Exp Med 210: 205-208, 2013

30. Li C, Shi C, Kim J, Chen Y, Ni S, Jiang L, Zheng C, Li D, Hou J, Taichman RS, et al: Erythropoietin promotes bone formation through EphrinB2/EphB4 signaling. J Dent Res 94: 455-463, 2015.

31. Ducy P, Zhang R, Geoffroy V, Ridall AL and Karsenty G: Osf2/Cbfa1: a transcriptional activator of osteoblast differentiation. Cell 89: 747-754, 1997.

32. Liu Y, Wang L, Kikuiri T, Akiyama K, Chen C, Xu X, Yang R, Chen W, Wang S and Shi S: Mesenchymal stem cell-based tissue regeneration is governed by recipient $\mathrm{T}$ lymphocytes via IFN- $\gamma$ and TNF- $\alpha$. Nat Med 17: 1594-1601, 2011.

33. Xu DJ, Zhao YZ, Wang J, He JW, Weng YG and Luo JY: Smads, p38 and ERK1/2 are involved in BMP9-induced osteogenic differentiation of C3H10T1/2 mesenchymal stem cells. BMB Rep 45: 247-252, 2012.

34. Zhao Y, Song T, Wang W, Wang J, He J, Wu N, Tang M, He B and Luo J: P38 and ERK1/2 MAPKs act in opposition to regulate BMP9-induced osteogenic differentiation of mesenchymal progenitor cells. PLoS One 7: e43383, 2012.

35. Wang Y, Ma J, Du Y, Miao J and Chen N: Human amnion-derived mesenchymal stem cells protect human bone marrow mesenchymal stem cells against oxidative stress-mediated dysfunction via ERK1/2 MAPK signaling. Mol Cells 39: 186-194, 2016.

36. Greenblatt MB, Shim JH, Zou W, Sitara D, Schweitzer M, Hu D, Lotinun S, Sano Y, Baron R, Park JM, et al: The p38 MAPK pathway is essential for skeletogenesis and bone homeostasis in mice. J Clin Invest 120: 2457-2473, 2010.

37. Higuchi C, Myoui A, Hashimoto N, Kuriyama K, Yoshioka K, Yoshikawa $\mathrm{H}$ and Itoh $\mathrm{K}$ : Continuous inhibition of MAPK signaling promotes the early osteoblastic differentiation and mineralization of the extracellular matrix. J Bone Miner Res 17: 1785-1794, 2002

38. Chang J, Sonoyama W, Wang Z, Jin Q, Zhang C, Krebsbach PH, Giannobile W, Shi S and Wang CY: Noncanonical Wnt-4 signaling enhances bone regeneration of mesenchymal stem cells in craniofacial defects through activation of p38 MAPK. J Biol Chem 282: 30938-30948, 2007

39. Ye G, Li C, Xiang X, Chen C, Zhang R, Yang X, Yu X, Wang J, Wang L, Shi Q, et al: Bone morphogenetic protein-9 induces PDLSCs osteogenic differentiation through the ERK and p38 signal pathways. Int J Med Sci 11: 1065-1072, 2014.

This work is licensed under a Creative Commons Attribution-NonCommercial-NoDerivatives 4.0 International (CC BY-NC-ND 4.0) License. 